\title{
Improving Energy Conversion Efficiency by means of Power Splitting in Dual Drive Train EV Applications
}

\author{
Michael A. Roscher, Roland Michel, and Wolfgang Leidholdt \\ imk automotive GmbH, Annaberger Straße 73, 09126 Chemnitz, Germany \\ Correspondence should be addressed to Michael A. Roscher; michael.roscher@rwth-aachen.de
}

Received 6 March 2013; Revised 7 July 2013; Accepted 21 July 2013

Academic Editor: Lingyang Song

Copyright ( 2013 Michael A. Roscher et al. This is an open access article distributed under the Creative Commons Attribution License, which permits unrestricted use, distribution, and reproduction in any medium, provided the original work is properly cited.

The limited amount of energy stored on board of battery electric vehicles (BEV) spurs research activities in the field of efficiency optimization for electric drive train applications in order to achieve an enhanced mileage. In this work a control method for BEV applications with two drive trains (e.g., one at the front and one at the rear axle) is presented. Herein, a simple optimization algorithm is introduced enabling to operate the two drives with different torque values, depending on the instantaneous operation point, leading to a reduction of apparent power losses on board. Simulations on a virtual BEV yield a decrease in the cumulated energy consumptions during typical BEV operation, leading to an increase in the achievable mileage.

\section{Introduction}

In recent years, the need for zero emission transportation spurred the broad market introduction of battery electric vehicles (BEV). BEVs can play a key role on the way to the environmentally conscious society [1]. Especially in mega cities where air pollution is a critical, the emission free vehicles can contribute to improve the health-related quality of life. Moreover, if the energy for battery charging comes from renewable sources (wind, solar, hydro power, etc.), the vehicles operate almost $100 \% \mathrm{CO}_{2}$ neutral.

However, one main drawback of battery powered vehicles is the very limited range, due to the very limited amount of energy stored in the battery. On the one hand, the efficiency of energy conversion of electric power trains is 2-3 times higher $(\eta>80 \%)$ than the efficiency of combustion engines $(\eta=20-$ $40 \%)$. On the other hand, the nowadays commercial battery cells comprise energy densities of maximum $250 \mathrm{Wh} / \mathrm{kg}$ [2], which is less than one fortieth of the theoretical energy density of conventional fuels (petrol gas approx. $12.000 \mathrm{Wh} / \mathrm{kg}$ ). Moreover, the energy density of common battery systems is reduced about almost one-half, due to efforts for housing, cooling, and integrated electrics/electronics.

An electric drive train substituting the combustion engine is one possible vehicle concept. Besides, various approaches (e.g., four separate in-wheel drives) are possible. In the following a vehicle architecture including two drives (i.e., one at the front and one at the rear axle ( $\rightarrow 4 \mathrm{WD})$; see Figure 1) is exemplarily considered.

In the illustrated $\mathrm{BEV}$ architecture the HVAC or at least the integrated compressor is supplied directly from the battery. To supply additional aggregates, the battery voltage is transferred into, for example, $12 \mathrm{~V}$ by a DC/DC converter. During vehicle acceleration and even speed, the drive train components (power converters and electric motors) convert the chemical energy stored in the battery in mechanical work. During regenerative breaking, the battery is recharged by the converters. The $4 \mathrm{WD}$ concept enables a very efficient recuperation, because regenerative breaking proceeds on both axles. Through incorporating a recuperative breaking, the possible range can be increased about up to several $10 \%$ $[3,4]$. However, the battery state-of-charge successively decreases during driving due to the apparent losses (wind, friction), differences in the level (track profile), and the energy consumption of the ancillary components [5].

From the literature several approaches are known referring to range prediction algorithms for HEV applications (e.g., in [6]) and similarly for BEVs [7] incorporation track profile information. Further, Zhang et al. [8] presented methods for driving range prediction of BEVs considering 


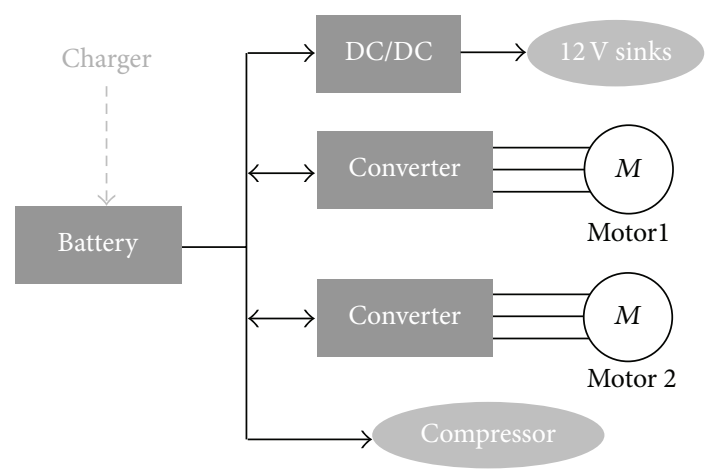

FIGURE 1: Power supply architecture of a two motor BEV concept.

environmental aspects (wind) and the driver's habits during driving, too. Besides, model based and expert systems specific energy management systems using stochastic approaches are known [9]. According to control strategies designed in order to optimize the energy efficiency on board of electrified vehicles, several references exist on the one hand focusing on managements systems of vehicles including at least two different energy storage systems (application with battery and supercap storage [10] or battery and fuel cell [11]) based on climatic, track profile, and operation point information.

On the other hand, energy saving strategies are known focusing on optimized control strategies for distinct vehicle components, for example, the air-conditioning system [12], by means of occasionally switching ancillary components according to the driving situation (battery temperature, power demand of the drive train, etc.). Optimizing the efficiency of electric motors in boost and regeneration operation is addressed in the literature references for HEV applications [13] and also HEV applications with two individual electric power trains in addition to the combustion engine, by optimizing the instantaneous operation points of the three individual drive trains [14]. Referring to the aforementioned approaches, a control strategy for the two-motor application in BEVs aiming an energy saving through optimized torque and speed control of the individual motors is presented in the following section.

\section{Power-Saving Operation}

In BEV applications with two individual power trains (on one axle or applied on two different axles), each of the power trains can be controlled independently [14]. During normal operation, the BEV's speed varies according to drive cycle and the driver's habits, but most of the time the speed can be assumed to remain almost constant over several seconds, except for sections of fast acceleration or regenerative breaking. During these periods of even speed with varied torque the overall mechanical power demand can be supplied by the both of the power trains or only by one power train, if possible. With only one motor supplying the torque (herein named individual operation), the electric power input can be reduced in comparison to two motors in synchronous operations, which is the cases if the power conversion with one motor is

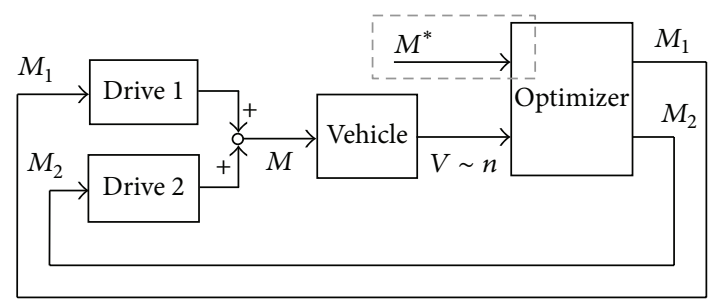

FIGURE 2: Control structure to optimize the energy consumption in a two motor EV power train concept.

more efficient, due to the magnetization and switching losses (idle losses) $[15,16]$. The instantaneous efficiency of power conversion of converter, and motor (electric to mechanical power, i.e., motor operation) depends on the rotation speed $n$ and the torque $M[17,18]$; for example, given with (1), where $P_{\text {mech }}$ is the mechanical power at the axles, $P_{\mathrm{el}}$ is the electric power input, $\eta_{\mathrm{pc}}$ is the actual efficiency of the power converter and $\eta_{\text {mot }}$ is the efficiency of the motor.

The general condition to meet in order to achieve the optimum efficiency is given with (2), where $M_{1}$ is the torque supplied from one of the drives (drive 1 ) and $M_{2}$ is the torque supplied from the other drive (drive 2). The overall torque supply $M$ correlates with the accelerator position operated by the vehicle driver as follows:

$$
\begin{aligned}
& \eta_{\mathrm{el}}=\frac{P_{\mathrm{mech}}}{P_{\mathrm{el}}}=\eta_{\mathrm{pc}} \cdot \eta_{\mathrm{mot}}=f(n, M), \\
& P_{\mathrm{el}}=\frac{n \cdot M_{1}}{\eta\left(n, M_{1}\right)}+\frac{n \cdot M_{2}}{\eta\left(n, M_{2}\right)} \stackrel{!}{=} \mathrm{Min} .
\end{aligned}
$$

A proposed control structure is illustrated in Figure 2. The overall motor torque demand $M^{*}$ (correlating with the accelerator position) and actual rotation speed $n$ (proportional to the vehicle's velocity $V$ ) are the input value for the optimizer unit.

From $M^{*}$ and $n$, the optimum set points $M_{1}$ and $M_{2}$ are derived, which are the torque control set point the both drives. The optimizer unit considers the efficiencies of the two possible operation cases, as stated above (1) and (2). The efficiencies in both cases, depending on the torque $M$ and the rotation speed $n$, are extracted form point maps $(\eta=$ $f\left(n, M^{*}\right)$ ), see (1) of the drive trains, using, for example, look-up tables or polynomials. Taking into account the actual efficiencies of the two drive trains in the two operation modes a distinction of cases is proposed according (3)

$$
\begin{aligned}
& M_{1}= \begin{cases}M^{*} & \text { for } \eta\left(n, M^{*}\right)>\eta\left(n, \frac{1}{2} M^{*}\right) \\
\frac{M^{*}}{2} & \text { for } \eta\left(n, M^{*}\right)<\eta\left(n, \frac{1}{2} M^{*}\right)\end{cases} \\
& M_{2}= \begin{cases}0 & \text { for } \eta\left(n, M^{*}\right)>\eta\left(n, \frac{1}{2} M^{*}\right) \\
\frac{M^{*}}{2} & \text { for } \eta\left(n, M^{*}\right)<\eta\left(n, \frac{1}{2} M^{*}\right) .\end{cases}
\end{aligned}
$$

Accordingly, both drive trains supply equal torques, that is, one-half of the overall torque demand $M^{*}$, if the efficiency 




(a)

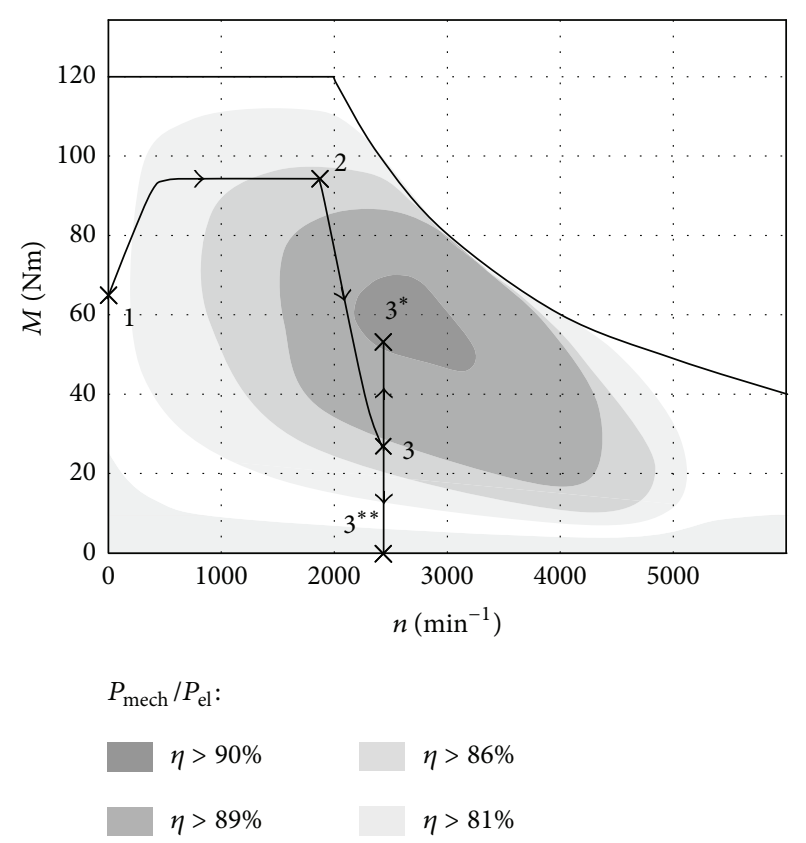

(b)

FIGURE 3: Comparison of the electric power demand at constant speed of two electric motors in synchronous and individual operation plotted versus the actual torque (a) and the possible trajectory for acceleration and subsequent even speed operation, with one drive train switched off after acceleration (b).

at $1 / 2 \cdot M^{*}$ is higher than the efficiency at $M^{*}$ (constant speed $n$ assumed). In the contrary case, the control set point of one motor is set to $M^{*}$, and the set point of the second motor is set to zero (i.e., turned off second drive train).

To elucidate a detailed control strategy of the drive trains itself (e.g., $[15,19])$, considering the variable battery voltage, temperature, load limitations and so forth, lies beyond the scope of this work and therefore is left out here.

With this approach the instantaneous power losses during even speed periods can be minimized. A comparison of both mentioned operation modes is illustrated in Figure 3(a), where the electric power effort is plotted over the instantaneous power at the drive side.

The dotted line indicates the power demand of two motors supplying the same torque. The blue solid line reflects the case where firstly one power train is active (the other converter is turned off) until the maximum power is reached. Towards higher torques, the second drive is active too (indicated in Figure 3(a) with turn on, motor number 2). At the point where the second drive is turned-on the power demand increases approximately about the amount of the idle losses. Obviously, at high total torque demand in case of operating the two power trains with different torques, the energy conversion is less efficient in comparison to two drives with equal torque operation. Therefore, an operation with both power trains supplying equal torques is to prefer (synchronous operation). But at lower torques the operation with one active motor can be more efficient, approximately until the saturation losses compensate the magnetization/swichting losses in idle running. The point where the efficiencies of synchronous and individual operations are equal is indicated in Figure 3(a) with "break even."

Hence, the intention is to operate with two active drive trains in high power demand operation and to switch-off one of the drives at low mechanical power demand, where the individual operation is more efficient. A possible trajectory during a driving situation with acceleration and even speed operation is depicted in Figure 3(b). Herein, the vehicle is accelerating (point $1 \rightarrow 2$ ). After accelerating greatly, the velocity is slightly increased for a certain period (point $2 \rightarrow 3$ ), and after that the vehicle speed is constant. In this operation (in the neighbourhood of point 3 in Figure 3(b)) it is more efficient to propel the axle with only one of the drives. Hence, one of the drives (converter and motor) is turned off, and two different operation points exist (points $3^{*}$ and $3^{* *}$ ). Due to the second motor being turned off, no magnetization losses are apparent in one motor, and no switching losses appear in one converter (point $3^{* *}$ ). In the shown exemplary case the overall efficiency is increased about approximately $1.3 \%$ at an electric power input of approximately $14.5 \mathrm{~kW}$. The instantaneous power saving is in a range of $180-200 \mathrm{~W}$.

The intention to turn-on and turn-off one of the drives, if it is preferable form energetic point of view, bears drawbacks during operation scenarios where the actual operation point lies in neighbourhood of the break-even point. In this case, on one hand it might be necessary to alternately switch between synchronous and individual operation, which can be detrimental in terms of mechanical stress on the components (gears, axles, etc.) and performance, due to the reaction time. On the other hand, the energy saving realized through 


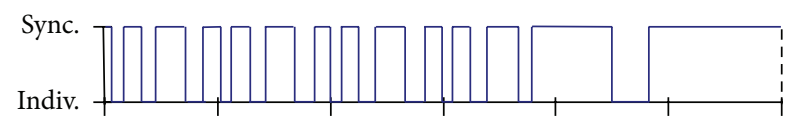

(a)



(b)

FIGURE 4: NEDC speed profile (b) and the periods where the drive trains operate in synchronous and individual operation (a).

operation mode switch is marginal in the close near of the break-even point, and therefore it is preferable to consider a locking function in order to avoid a switch from synchronous and individual mode too often in the mentioned case. A possible solution would be a hysteresis two-step control based on the efficiency values or the actual power saving which can be calculated instantaneously. Another approach includes a time based locking function to avoid the switch between the modes for certain periods.

The outlined strategy ( $\eta$-optimization with locking function) is easy to be implemented in a control unit of a BEV. Simulation results carried out in use of the presented method are given and discussed in the following section.

\section{Simulation and Discussion}

The proposed approach to optimize the efficiency of power conversion of a battery electric vehicle is implemented in the control strategy of a virtual BEV in MATLAB/Simulink. Herein, the model of the BEV includes two individual drives (including PSM drives with $25 \mathrm{~kW}$ nominal power each) referring to Figure 1, where both motors are mechanically connected to one and the same differential gear at the rear axle. By this way the both drives always operate with equal speed and the torques add. In order to avoid the excessive switching from synchronous to individual operation and vice versa, a time based lock is included (lock duration $=5 \mathrm{~s}$ ). The time constant for locking was chosen in accordance with the reaction time of the power train components. If the power demand exceeds the power which can be applied from one drive (compare to Figure 3(a)), the locking function is neglected, and the second drive instantly becomes active.

The incorporated BEV model includes a $20 \mathrm{kWh}$ lithiumion-based battery, and the total weight of the vehicle is assumed to be $1100 \mathrm{~kg}$ with a drag coefficient $c_{d}=0.28$ (front cross section area $2.5 \mathrm{~m}^{2}$ ), coefficient of rolling friction $c_{R}=$ 0.025 . For validation various standardized drive cycles were taken into account. In the following the results from simulations incorporating the common NECD ("New European Drive Cycle") load cycle are illustrated in detail (preferable the NEDC reflects highway and urban use; investigations on
TABLE 1: Energy consumption during one NEDC cycle and corresponding maximum range.

\begin{tabular}{cccc}
\hline Operation & $1 \times$ NEDC & $100 \mathrm{~km}(\mathrm{NEDC})$ & max. range $(20 \mathrm{kWh})$ \\
\hline sync./indiv. & $1.567 \mathrm{kWh}$ & $14.22 \mathrm{kWh}$ & $140.6 \mathrm{~km}$ \\
only sync. & $1.578 \mathrm{kWh}$ & $14.32 \mathrm{kWh}$ & $139.7 \mathrm{~km}$ \\
\hline
\end{tabular}

different load cases yield similar results and therefore are left out here). The speed profile of the NEDC is depicted in Figure 4(b). One NEDC tasks 1180 seconds with a maximum speed of $120 \mathrm{~km} / \mathrm{h}$ and the covered distance is approximately $11 \mathrm{~km}$.

Besides the speed profile also the periods where both motors are active (sync.) and the periods where only one drive is operating (indiv.) are illustrated in Figure 4(a). Obviously, most of the time both drive trains are active. The individual operation mainly occurs during low speed driving with almost constant speed. The electric energy demands are given in Table 1, where a comparison is given between the modes with event-oriented operation in individual and synchronous operations (sync./indiv.) and an operation where the two drives always supply the same torque (only sync.). The given values refer to the energy consumption during one NEDC cycle, the electric energy required for $100 \mathrm{~km}$ mileage under NEDC load and the theoretical range under NEDC load achievable with a fully charged $20 \mathrm{kWh}$ Li-ion battery (additional components are assumed to have a total power consumption of $200 \mathrm{~W}$ ).

Noticeably, the energy consumption of the BEV model incorporating the proposed control strategy is reduced in comparison to an operation without splitting of torque. As visible in Figure 4, the individual mode is active mainly in sections with low, even speed, that is, low/moderate power demand. Even if the energy saving during low power operation is in a range of several tens or up to a few hundreds Watt, the saved energy cumulates over longer driving periods. The relative energy saving for the investigated case is approximately $0.6 \%$. This value does not seem to be very much, but the energy can be saved without any hardware effort, only by using the proposed control strategy. Anyhow, the saving of 
$0.6 \%$ of the consumed energy extends the maximum range in the presented case about almost $1 \mathrm{~km}$ or is equal to a weight reduction of the vehicle about approximately $12 \mathrm{~kg}$, respectively. Furthermore, in the investigated case two similar drives are considered, including two converters and two motors. A further energy saving may be possible by using drives optimized for different ranges of operation. This way the break even point can be shifted towards higher power values, and the amount of saved energy can be increased. In case of using two individual current-excited motors, the relative energy saving can be increased due to the higher idle losses. Herein, during individual operation the rotor losses of one motor can be eliminated completely.

However, the presented investigation results are carried out on a BEV simulation model and therefore may differ from results obtained from tests conducted on real vehicles. Hence, the implementation of the proposed methods in the energy management system of a BEV and the subsequent evaluations on vehicle handling, performance, and energy consumption are challenging topics of our further research and development efforts. Moreover, the application of two (or more) drives at different axles may be more critical and needs detailed investigations concerning the vehicle handling and performance in case of torque splitting among the two axles, and therefore those investigations are also a topic of future work.

\section{Conclusion}

This work focuses on the optimization of power conversion on board of a battery electric vehicle including two drive trains. Due to the characteristics of the drive trains in case of low or moderate constant speed with varying torque operating, only one drive instead of two active drive trains may yield a higher efficiency in power conversion. Accordingly, a control structure is derived and is implemented in the energy management control unit of a virtual BEV. During operation (i.e., NEDC load) a reduction of energy consumption about $0.6 \%$ can be achieved without any additional hardware effort. This energy saving corresponds to a reduction of the vehicle's weight about approximately $12 \mathrm{~kg}$.

\section{References}

[1] M. A. Roscher, J. Vetter, and D. U. Sauer, "Influence of cathodes technology on the power capability and charge acceptance of lithium ion batteries," in Proceedings of the 24th International Battery, Hybrid and Fuel Cell Electric Vehicle Symposium (EVS '09), Stavanger, Norway, May 2009.

[2] Panasonic, "NNP series-NCR18650A," Technical Datasheet, 2010, http://www.panasonic.com/.

[3] K. Imai, T. Ashida, Y. Zhang, and S. Minami, "Theoretical performance of EV range extender compared with plugin hybrid," Journal of Asian Electric Vehicles, vol. 6, no. 2, pp. 1181-1184, 2008.

[4] Y. Yang, J. Liu, and T. Hu, "An energy management system for a directly-driven electric scooter," Energy Conversion and Management, vol. 52, no. 1, pp. 621-629, 2011.

[5] Y. Ota, H. Taniguchi, T. Nakajima, K. M. Liyanage, J. Baba, and A. Yokoyama, "Autonomous distributed V2G (vehicle-to-grid) considering charging request and battery condition," in Proceedings of the IEEE PES Innovative Smart Grid Technologies Conference Europe (ISGT Europe '10), November 2010.

[6] S. Kermani, S. Delprat, R. Trigui, and T. Guerra, "Predictive energy management of hybrid vehicle," in Proceedings of the IEEE Vehicle Power and Propulsion Conference (VPPC '08), vol. 20, no. 1, pp. 1-6, September 2008.

[7] A. Dardanelli, M. Tanelli, S. M. Savaresi, and M. Santucci, "Active energy management of electric vehicles with cartographic data," in Proceedings of the IEEE International Electric Vehicle Conference (IEVC '12), Greenville, SC, USA, March 2012.

[8] Y. Zhang, W. Wang, Y. Kobayashi, and K. Shirai, "Remaining driving range estimation of electric vehicle," in Proceedings of the IEEE International Electric Vehicle Conference (IEVC '12), Greenville, SC, USA, March 2012.

[9] C. Dextreit, F. Assadian, I. Kolmanovsky, J. Mahtani, and K. Burnham, "Hybrid electric vehicle energy management using gametheory," SAE Technical Paper 2008-01-1317, 2008.

[10] A. A. Ferreira, J. A. Pomilio, G. Spiazzi, and L. de Araujo Silva, "Energy management fuzzy logic supervisory for electric vehicle power supplies system," IEEE Transactions on Power Electronics, vol. 23, no. 1, pp. 107-115, 2008.

[11] J. Schiffer, O. Bohlen, R. W. De Doncker, D. U. Sauer, and K. Y. Ahn, "Optimized energy management for fuelcell-supercap hybrid electric vehicles," in Proceedings of the IEEE Vehicle Power and Propulsion Conference (VPPC '05), pp. 716-723, September 2005.

[12] M. A. Roscher, W. Leidholdt, and J. Trepte, "High efficiency energy management in BEV applications," International Journal of Electrical Power and Energy Systems, vol. 37, no. 1, pp. 126-130, 2012.

[13] H. Wu, L. Li, B. Kou, and Z. Ping, "The research on energy regeneration of permanent magnet synchronous motor used for hybrid electric vehicle," in Proceedings of the IEEE Vehicle Power and Propulsion Conference (VPPC'08), pp. 1-4, September 2008.

[14] L. Jishun, L. Jun, W. Qingnian, W. Jiaxue, and S. Jinhu, "Study on mechanism of energy saving for double motor configuration hybrid electric vehicle," in Proceedings of the International Conference on Mechatronic Science, Electric Engineering and Computer (MEC '11), pp. 2596-2602, August 2011.

[15] J. Lee, K. Nam, S. Choi, and S. Kwon, "A lookup table based loss minimizing control for FCEV permanent magnet synchronous motors," in Proceedings of the IEEE Vehicle Power and Propulsion Conference (VPPC '07), pp. 175-179, September 2007.

[16] J. J. C. Gyselinck, L. Vandevelde, D. Makaveev, and J. A. A. Melkebeek, "Calculation of no load losses in an induction motor using an inverse vector Preisach model and an eddy current loss model," IEEE Transactions on Magnetics, vol. 36, no. 4, pp. 856860, 2002.

[17] B. J. Chalmers and I. Musaba, "Performance characteristics of permanent-magnet and reluctance machines to meet EV requirements," in Proceedings of the IEE Colloquium on Machines and Drives for Electric and Hybrid Vehicles, Digest No: 1996/152, August 2002.

[18] F. Deng, "Improved analytical modeling of commutation losses including space harmonic effects in permanent magnet in brushless DC motors," in Proceedings of the IEEE International Electric Machines and Drives Conference (IEMDC'97), May 1997.

[19] J. Lee, K. Nam, S. Choi, and S. Kwon, "Loss-minimizing control of PMSM with the use of polynomial approximations," IEEE Transactions on Power Electronics, vol. 24, no. 4, pp. 1071-1082, 2009. 

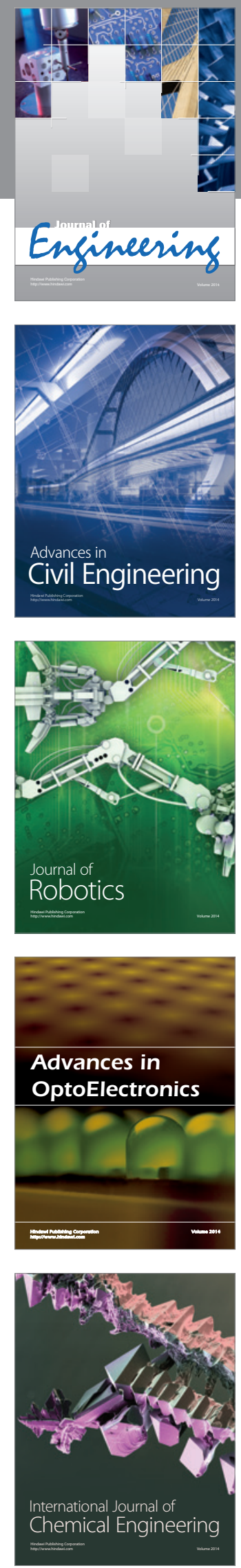

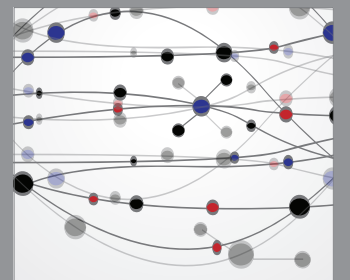

The Scientific World Journal
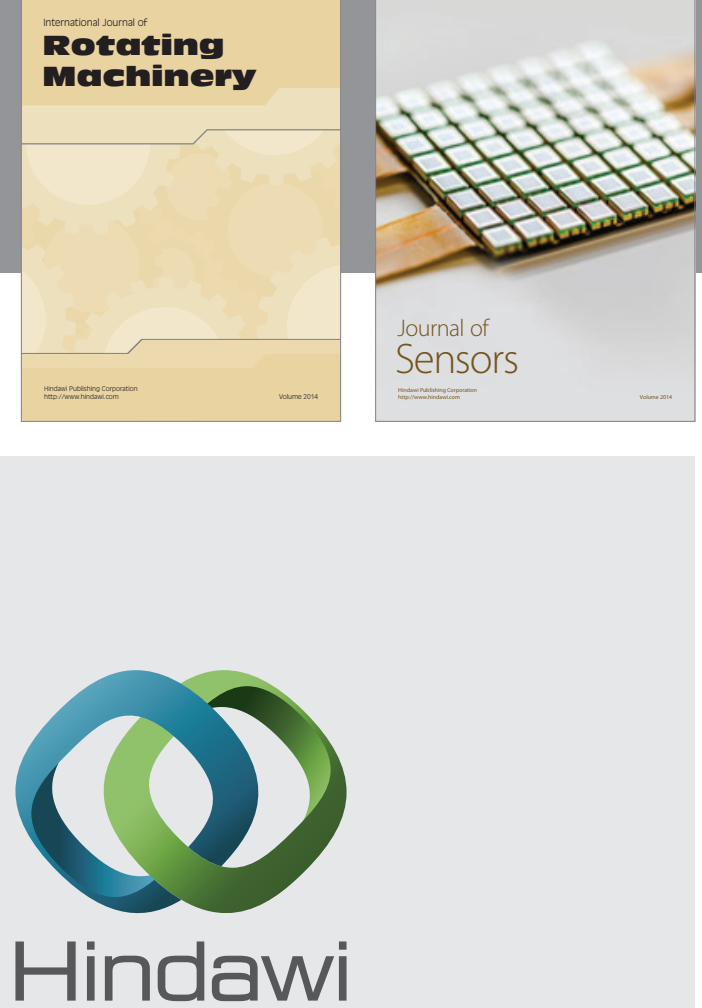

Submit your manuscripts at http://www.hindawi.com
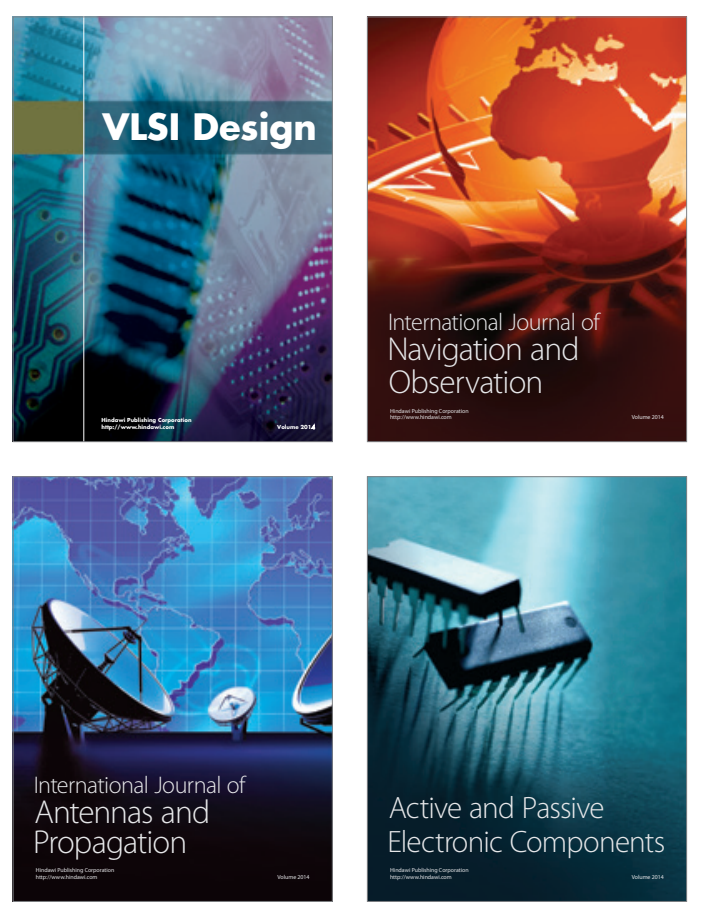
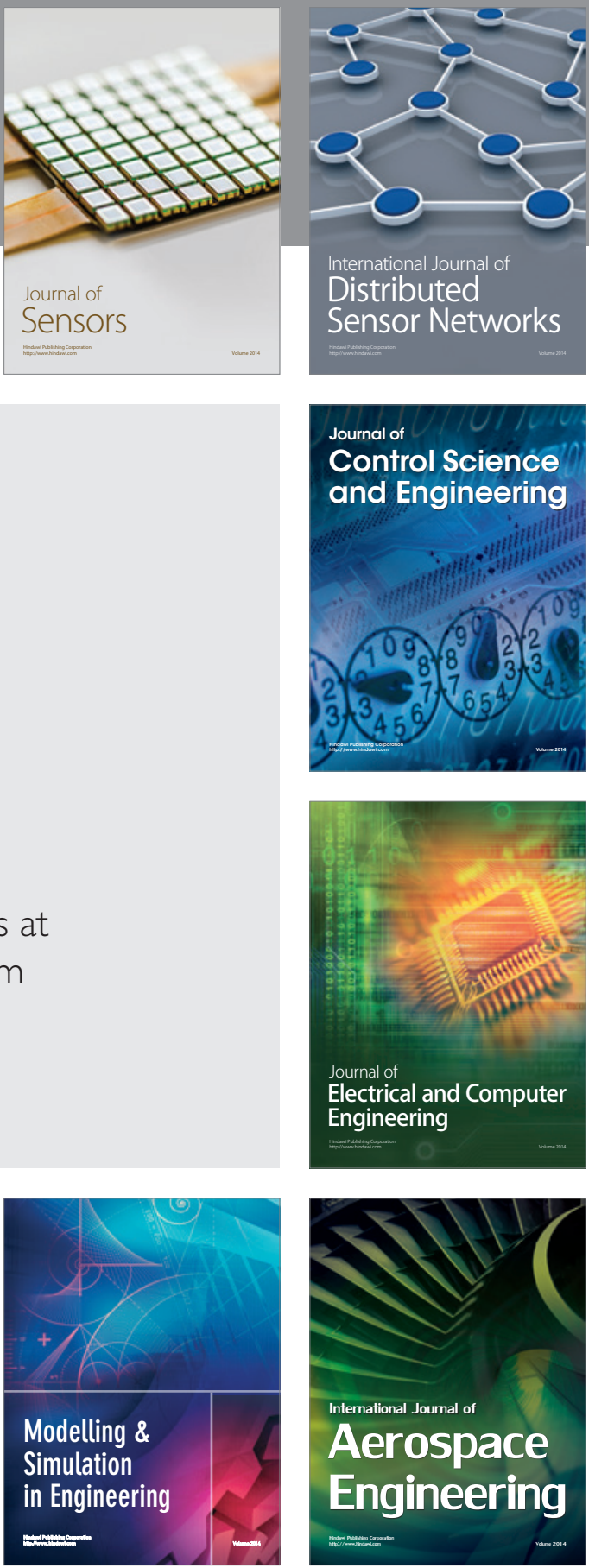

Journal of

Control Science

and Engineering
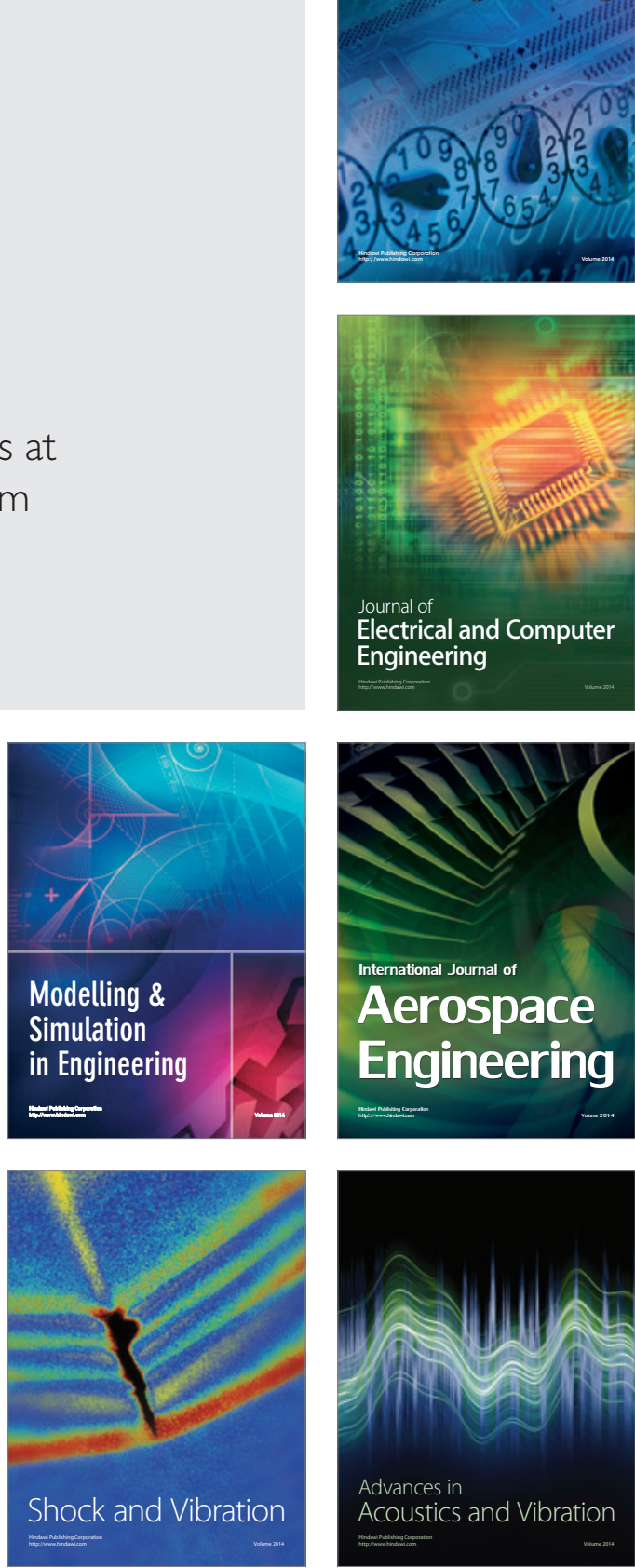